\title{
IdeAs
}

Idées d'Amériques

$14 \mid 2019$

Populismes dans les Amériques

\section{Jana Riess, The Next Mormons. How Millennials Are Changing the LDS Church}

New York, Oxford University Press, 2019, 312 pages

\section{Blandine Chelini-Pont}

\section{(2) OpenEdition}

\section{Journals}

Édition électronique

URL : http://journals.openedition.org/ideas/6354

DOI : $10.4000 /$ ideas.6354

ISSN : 1950-5701

Éditeur

Institut des Amériques

\section{Référence électronique}

Blandine Chelini-Pont, « Jana Riess, The Next Mormons. How Millennials Are Changing the LDS Church », IdeAs [En ligne], 14 | 2019, mis en ligne le 01 octobre 2019, consulté le 24 septembre 2020. URL : http://journals.openedition.org/ideas/6354 ; DOI : https://doi.org/10.4000/ideas.6354

Ce document a été généré automatiquement le 24 septembre 2020.

\section{$(1) \Theta \Theta$}

IdeAs - Idées d'Amériques est mis à disposition selon les termes de la licence Creative Commons Attribution - Pas d'Utilisation Commerciale - Pas de Modification 4.0 International. 


\title{
Jana Riess, The Next Mormons. How Millennials Are Changing the LDS Church
}

New York, Oxford University Press, 2019, 312 pages

\author{
Blandine Chelini-Pont
}

\section{RÉFÉRENCE}

Jana Riess, The Next Mormons. How Millennials Are Changing the LDS Church, New York, Oxford University Press, 2019, 312 pages

1 Jana Riess assure qu'elle n'a eu aucune prise sur la couverture de son nouveau livre. On y voit, sur un fond noir, deux pouces visiblement féminins, aux ongles peints en noir, tenant ouverte une Bible aux pages immaculées et à la couverture rose... Si jamais couverture avait tenté de capter l'essence de son contenant, alors celle-ci discrètement accrocheuse hits the bull's eye. Le cœur de l'ouvrage se veut quelque peu décapant et a provoqué une mini-tempête au sein des études mormones tout comme l'intérêt des sociologues du religieux.

2 Titulaire d'un doctorat en histoire religieuse américaine de l'université Columbia, lectrice-éditrice de profession au Publishers Weekly et éditorialiste du site web Religion News Service, Jane Riess est connue pour sa participation à plusieurs livres, certains de facture académique sur l'Église des Saints du Dernier Jour, comme Mormonism and American Politics (avec Randall Balmer, Columbia University Press, 2015), d'autres plus convictionnels, voire pastoraux - Jane Riess souhaitait être pasteur(e) dans sa jeunesse avant de se convertir au mormonisme - comme Flunking Sainthood Every Day en 2015 et, plus récemment, The Prayer Wheel: Rediscovering Prayer with an Ancient Spiritual Practice, deux ouvrages marqués par une forme de décalage et d'originalité inattendue. Dans ce nouveau livre, à la visée académique, Jana Riess a travaillé en duo avec Benjamin Knoll, professeur associé de sciences politiques au John Marshall Harlan Centre de Danville 
College, dans le Kentucky. Ce dernier s'intéresse à la relation entre opinion publique et comportement électoral, entre religion, race et politique. Ces recherches l'ont amené à commenter l'actualité de l'Église mormone, à laquelle il a consacré de nombreux articles de vulgarisation sur la Toile.

3 La thèse principale de cet ouvrage est que la génération née dans les années 1980 et 1990 - les American Millennials - a quitté la religion mormone dans une proportion sans précédent. Pendant longtemps, l'Église de Jésus-Christ des Saints des Derniers Jours avait été une exception : près des trois quarts des personnes qui grandissaient en «mormonie » restaient dans cette mouvance jusqu'à leur mort. Dans The Next Mormons, Jana Riess démontre que cet état de chose connaît une évolution sans précédent.

Riess et Knoll se sont appuyés sur une étude nationale portant sur quatre générations de mormons confessants et d'anciens mormons, ainsi que sur des entrevues personnelles, outre la réutilisation de plusieurs sondages récents réalisés par Pew, Gallup, l'enquête Peculiar People que David Campbell a menée il y a cinq ans, l'enquête sociale générale American National Elections Survey, l'Understanding Mormon Disbelief Survey (2011) et le Mormon Gender Issues Survey (2015). Grâce au savoir-faire analytique de Knoll, Riess a exploré les croyances et les comportements religieux des jeunes adultes mormons contemporains et constaté que, même si le niveau de croyance demeure élevé (Partie 1: Foundations), leur loyauté institutionnelle est moins certaine que celle de leurs parents et grands-parents. Pour un nombre croissant de Millennials, les tensions entre les idéaux stricts de l'Église et l'ingestion par leur génération de l'individualisme et du pluralisme s'avèrent trop fortes, ce qui les pousse à quitter la foi - souvent en proie à une profonde angoisse personnelle (Partie 3 : Passages of Faith and Doubt). Ceux qui restent dans le giron de l'Église tentent d'équilibrer soigneusement l'importance sacrée que celle-ci accorde à la famille traditionnelle, avec une tolérance plus inclusive, propre à leur génération, envers les couples homosexuels et... l'égalité des femmes. Car les familles mormones changent aussi. Un nombre plus grand de mormons restent célibataires (y compris les femmes qui font des études plus longues), davantage de jeunes parents ont moins d'enfants et de plus en plus de femmes travaillent à l'extérieur du foyer (Partie 2: Changing Definition of Family and Culture).

Ce grand travail d'enquête se laisse appréhender au moins de deux façons.

6 La première revient à abonder dans le sens des conclusions proposées par les auteurs et de déduire raisonnablement que les Mormons contemporains ne sont pas épargnés, à leur tour, par la sécularisation, l'individuation du croire et le nouvel égalitarisme sexuel des droits civils. Malgré leur très majoritaire orthopraxie, malgré leur fort sentiment communautaire et leur culture familialiste, ils n'échappent pas aux débats et évolutions sociétales qui touchent la société américaine. Il est également possible de considérer la plus grande perméabilité des membres de l'Église mormone avec l'extérieur par l'effet même du brassage des mormons. Même si l'Église des Saints des derniers jours est toute puissante au sein de l'Utah, cet état protège strictement la liberté religieuse, y compris celle des mormons en leur for interne, comme il protège leurs autres libertés. L'Église n'a aucune autre prise directe - hormis l'excommunication du croyant qui n'est plus si courante - sur la liberté de mouvement et de pensée de ses fidèles. L'Utah connaît une forte immigration et pluralisation religieuse, ainsi qu'une diversité croissante de comportements. À l'inverse, L'Église regroupe une population qui a fini depuis une génération par excéder largement celle de son berceau d'origine sous l'effet de la mobilité - les mormons s'installent, comme 
d'aucuns, là où le bon vent économique et leur esprit d'entreprise les poussent - et de la conversion missionnaire - la mission fait partie intégrante des obligations spirituelles. La communauté mormone s'éparpille donc aux États-Unis et au Canada, et jusqu'en Allemagne. Elle grandit dans les pays étrangers - elle aurait dépassé les 10 millions au Mexique. Les mormons font l'expérience de leur singularité au milieu d'une population qui n'est pas comme eux et cette interaction permanente provoque des ajustements de leur sentiment élitaire et de leur foi en la sainte forme de la famille nucléaire. Ainsi, des phénomènes auparavant plus marginaux, comme le travail des femmes, la tolérance envers les homosexuels et surtout la liberté d'entrer et de sortir de cette Église sont devenus beaucoup plus courants et pourront caractériser de plus en plus les générations montantes.

7 La seconde lecture possible est plus réservée quant à la présentation même du discours narratif qui sous-tend la présentation de ces évolutions. Ce dernier met en avant, sans beaucoup de nuance et avec une empathie non dissimulée, un jugement systématiquement compréhensif envers les sortants et les dissidents qui iraient dans le sens de l'histoire et systématiquement critique envers une institution et une proposition de foi qui contraindrait ses membres, les feraient souffrir et serait désormais en passe de devenir anachronique. Or, que l'on pose le discours de justification des uns et l'état de la doctrine de l'autre revient à établir un fait descriptif et l'on s'attendrait à ce que l'écriture de la description en reste à cette distance. L'écart se creuse entre les pratiques personnelles et la subjectivité des jeunes mormons, il se creuse entre la réalité quotidienne des personnes ou des familles et la normativité dogmatique mormone en matière de complémentarité sexuelle et de sainte cellule familiale. C'est un autre fait certain. Le fait que la rupture survienne et augmente pourrait tout aussi bien suggérer que cette Église n'est pas une secte étouffante et que sa pression sur les consciences est finalement moins forte que l'on pourrait se l'imaginer. Comme affirmer dans le même temps que de plus en plus de jeunes quittent cette Église et sa foi parce qu'elles n'évoluent pas et que les membres à l'intérieur de cette Église sont en train d'évoluer ? Outre qu'il est difficile d'être absolument certain de la représentativité d'une enquête finalement assez modeste ${ }^{1}-$ nonobstant l'immense travail des auteurs - peut-être manque-t-il, pour vraiment équilibrer ce tableau et nous donner toutes les clefs pour comprendre la situation actuelle au sein de l'Église des Saints des derniers jours, une présentation de sa régulation doctrinale et de ses potentialités « canoniques » de transformation? Cette Église a évolué dans le passé : sa position originelle sur la polygamie, sa position originelle sur la non-prêtrise des descendants de Caïn (les noirs), sa position sur les malédictions divines à l'écart de certains peuples ont été abolies. Elle tolère le divorce. Comment se décident ces changements, quelles sont les modalités possibles, sont-ils mis en valeur ou au contraire esquivés ? Les changements ont-ils lieu à cause de l'extérieur, sous la pression de la loi civile, la jurisprudence fédérale, les besoins missionnaires, l'état de la société ? Toutes ces questions cruciales auraient pu (ou dû) être intégrées dans l'élaboration des conclusions de cet ouvrage, par ailleurs très documenté. 


\section{NOTES}

1. Cette enquête faite en 2016, présentée à la fin de l'ouvrage a été menée et analysée par Benjamin Kroll et Jane Riess, s'est appuyée sur un ratio de 1156 mormons et 540 ex-mormons et 63 entretiens, la plupart (sans précision de chiffres) nés dans une famille mormone.

\section{AUTEURS}

\section{BLANDINE CHELINI-PONT}

Blandine Chelini-Pont est professeur d'histoire contemporaine est membre du Laboratoire Interdisciplinaire de Droit des Médias et des Mutations Sociales (LID2MS) à Aix-Marseille Université. Elle est également membre associée au Groupe Sociologie des Religions et de la Laïcité, EPHE-CNRS. blandine.chelini-pont@univ-amu.fr 